\title{
Wie Familienfragen öffentlich werden
}

\section{Der Berliner Familienbericht 2010 als Beispiel}

\author{
Peter Ruhenstroth-Bauer
}

Rechtsanwalt Peter Ruhenstroth-Bauer ist seit 2006 in der Kommunikationsberatung tätig. Zuvor war er als Hörfunkjournalist, ab 1998 als Stellvertretender Chef des Presse- und Informationsamtes der Bundesregierung und in der 15. Legislaturperiode als Staatssekretär im Bundesministerium für Familie, Senioren, Frauen und Jugend tätig. Im Ehrenamt ist er Vorsitzender des Berliner Beirats für Familienfragen. Internet http://www.

KommunikationundStrategie.de
Familienfreundlichkeit entscheidet sich vor Ort, in den Kommunen, in den Stadtteilen und damit im unmittelbaren Lebensumfeld der Familien. Wie geht es den Familien dort, welche Bedingungen finden sie für ibre unterschiedlichen Lebensrealitäten vor, mit welchen Hindernissen haben sie im Alltag zu kämpfen?

Antworten auf diese Fragen, Daten zu den verschiedenen Familientypen und Handlungsfelder für eine nachhaltig orientierte Familienpolitik finden sich in der Familienberichterstattung.

Der Berliner Senat ist für den Berliner Familienbericht 2010 einen neuen und bisher einmaligen Weg gegangen: Der »Berliner Beirat für Familienfragen « wurde 2007 mit der Erarbeitung des nächsten Familienberichts beauftragt. Das Besondere an dem Gremium ist seine Vielfalt: Neben vielen anderen Vertreterinnen und Vertretern aus Verbänden, Kirchen und Wohlfahrtsorganisationen sind auch alle Parteien des Berliner Abgeordnetenhauses sowie die Wirtschaft vertreten.

\section{Der Berliner Beirat für Familienfragen}

Ende 2007 hat der Berliner Senat den Berliner Beirat für Familienfragen, ein überparteiliches und ehrenamtliches Beratungsgremium, berufen. Mit der Ernennung der 22 Vertreterinnen und Vertreter des Gremiums aus Wissenschaft, Wirtschaft, Gewerkschaften, Politik, Wohlfahrt, Glaubensgemeinschaften und Einzelpersonen wurde der Familienpolitik im Land Berlin ein sichtbar neuer Stellenwert gegeben. Denn in dem Gremium sitzen nicht nur Vertreterinnen aller Fraktionen des Berliner Abgeordnetenhauses, sondern auch solche Akteure, die traditionell nicht mit Familienpolitik in Verbindung gebracht werden, so zum Beispiel die Handwerkskammer oder die Industrie- und Handelskammer Berlins. Fragen und Probleme bei der Ver- einbarkeit von Familie und Beruf gehören deshalb selbstverständlich zu den zentralen Themenfeldern, mit denen sich der Berliner Beirat für Familienfragen beschäftigt.

Aber nicht nur die Zusammensetzung, auch die vom Senat beschlossene Aufgabenstellung in der Satzung des Familienbeirats zeigen, dass die Politik bereit scheint, beim Familienthema ein breites Kompetenzfeld zu aktivieren: Der Beirat ist aufgefordert, familienrelevante Senatsentscheidungen vorab beratend zu begleiten (1), Familienfragen in der Stadt zu popularisieren und schließlich: den Familienbericht des Landes Berlin in dieser Legislaturperiode zu erarbeiten. Ende 2010 soll nun der Familienbericht für diese Legislaturperiode abgeschlossen sein und dem Regierenden Bürgermeister übergeben werden.

Der Berliner Familienbeirat hat in seiner Zielsetzung für den Familienbericht klare Ziele formuliert. Neben einer Bestands- und Bedarfsanalyse der familienorientierten Angebote Berlins soll auch eine Weiterentwicklung und gegebenenfalls Neukonzeption familienpolitischer Maßnahmen angeboten werden.

Für die vielen verschiedenen Akteure der Hauptstadt werden so Angebote und Beispiele für eine unmittelbare Umsetzung zusammengestellt. Im Ergebnis wird der Berliner Familienbericht also nicht nur Risiken beschreiben, sondern auch Chancen aufzeigen: neben den »Defiziten « wird ein gleichberechtigter Schwerpunkt auf bereits bestehende gute Praxis in Berlin gelegt. Denn dass der dringende Bedarf, die Aktivitäten in den zwölf Berliner Bezirken zu vernetzen, besteht, zeigt schon ein oberflächlicher Blick in die Bezirke: Die Beispiele guter Praxis kommen in der Hauptstadt über manche Bezirksgrenze nicht hinaus.

\section{Das sind die Inhalte}

Um die Inhalte des Familienberichts zu konturieren, wurden von den Beiratsmitgliedern 2008 in einer Prioritätsabfrage 
sechs Schwerpunktthemen des Familien-

berichts identifiziert:

- Familien und Beruf vereinbaren, Work-Life-Balance

- familienfreundliche Regionen und Stadtquartiere

- Ausbau der familienfreundlichen Infrastruktur in Berlin

- Damit Familienarmut nicht zum Alltag gehört!

- Bildung und Ausbildung für alle Kinder und Jugendlichen

- Integration in unserer Stadt - als Querschnittsthema für alle vorhergehenden Schwerpunktthemen

\section{Partizipation als Richtschnur}

Bei der Erarbeitung dieser Themen darf es nach Auffassung des Familienbeirats nicht allein um die Expertise der Wissenschaft, der Akteure für Familien in der Stadt gehen. Es geht vor allem auch um die Anregungen und Hinweise der Familien selbst. So wurde der Satz »Der Berliner Familienbericht wird nicht über $\mathrm{Fa}$ milien, sondern mit den Familien Berlins erarbeitet « zur Richtschnur für die Berichtserarbeitung.

Gleichzeitig sollten die familienpolitischen Akteure wie die Öffentlichkeit die Ergebnisse der verschiedenen inhaltlichen Schwerpunkte nicht erst mit der Übergabe des Berichtes kennenlernen. Deshalb wird die Berichterarbeitung vom Familienbeirat seit 2008 als ein öffentlicher Prozess verstanden. Nach jedem Kapitel werden die Zwischenergebnisse öffentlich kommuniziert und geben so Anlass zur Diskussion und gleichzeitig auch schon während der Berichtserarbeitung zur politischen Reaktion.

Die Themenschwerpunkte werden dabei in gleicher Struktur mit unschiedlichen Instrumenten bearbeitet. Dazu gehören die vier verschiedenen Herangehensweisen und Arbeitsformen (2):

- Beteiligung

- Praxisorientierung

- wissenschaftliche Expertise

- Diskussionsveranstaltungen und Fachforen

Nach einer Einführung in die verschiedenen Themen und der Bedeutung für die Familien in Berlin wird jeweils mit einer eigenen Bestandsaufnahme beschrieben, wie die Wirkungen der aktuellen Angebote, Maßnahmen und Strukturen auf

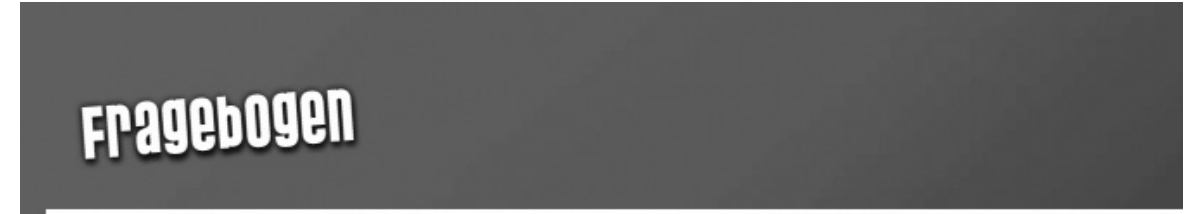

1. In welchem Berliner Klez sind Sie zu Hause?

2. Welche Angebote (Freizeitmöglichkeiten, Wohnumfeld, Nachbarschaftszentren etc.) schätzen Sle besonders in Ihrem Klez?

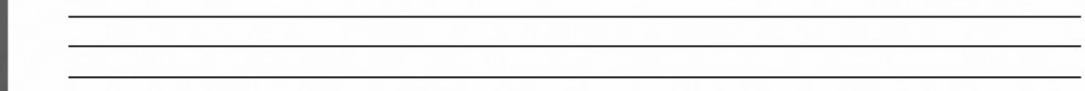

3. Was würden Sie in Ihrem Kiez für das Zusammenleben noch verbessern?

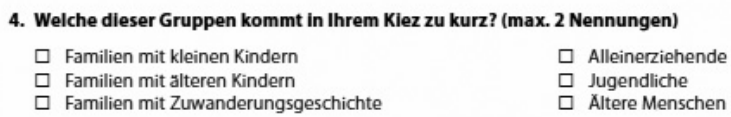

Familien mit Jugendliche

5. Wie beurteilen Sie das Zusammenleben der verschledenen Generationen in Ihrem Kiez?

$\begin{array}{ll}\square \text { gut } & \square \text { schlecht } \\ \square \text { weniger gut } & \square \text { keine Meinung }\end{array}$

6. Haben Sle Vorschläge, wie und wo der Kontakt zwischen alt und jung in Ihrem Klez stärker gefördert werden könnte?

7. Welche drel Dinge würden Sle zuerst angehen, wenn es darum geht, die Stadt Berlin famillienfreundlicher zu gestalten?
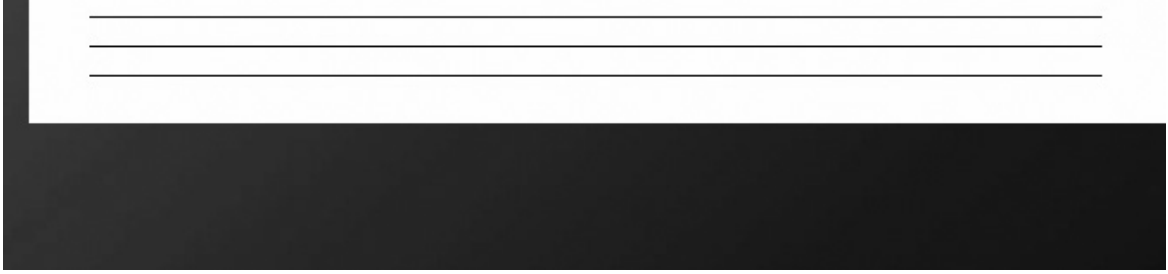

Der Berliner Familienbericht 2010 wird mit breiter Beteiligung von Bevölkerung und Organisationen in der Hauptstadt erstellt. So werden in einer begleitenden Kampagne die Bewohner gefragt, was an Verbesserungen in ihrem Stadtteil notwendig ist und was jetzt schon so gut läuft, dass andere es nachahmen können.

Internet http://www.familienbeirat-berlin.delfamilienbericht.html

Familien aussehen. Die wissenschaftliche Bewertung bildet die Grundlage für die Analyse, aus der sich dann in der folgenden Diskussion eine Prioritätensetzung mit Handlungsansätzen und Vorschlägen zur Weiterentwicklung ergibt. So entwickelt der Familienbericht als Beteiligungsprojekt mit Familien, Kindern und Jugendlichen, Seniorinnen und Senioren und der Fachöffentlichkeit aus der Gesamtschau der unterschiedlichen Perspektiven Umsetzungsempfehlungen, die die Akteure unter Einbeziehung schon beste- hender guter Praxis aus Berlin als Anregung und Auftrag verstehen sollten.

\section{Verschiedene Beteiligungs- instrumente ergeben ein Bild}

Damit die breite Beteiligung an den Schwerpunkten des Familienberichts erreicht werden konnte, wurde 2008 zum ersten Mal ein Online-Dialog (3) durchgeführt (Internet http://www.Zusammenleben-inBerlin.de). Vier Wochen lang haben die 
Berlinerinnen und Berliner ihre eigenen Schwerpunkte entwickelt, Themen diskutiert und Vorschläge gemacht. Wie bei vielen Online-Diskussion gab es einige, die als registrierte Teilnehmer intensiv diskutiert haben und viele, die mitgelesen haben. Unterstützt durch prominente Paten des Dialogs, Schauspieler, TV-Journalistinnen, Sportler und andere stadtbekannte Einzelpersönlichkeiten diskutierten 318 registrierte Nutzerinnen und Nutzer; hinzu kamen 2.816 individuelle Mitleser. Mit insgesamt 38.000 Seitenzugriffen war die Nutzungsund Reichweitenbilanz des ersten OnlineDialogs ein Erfolg. Bei einem hohen Anteil männlicher Teilnehmer $(35 \%)$ und einem Schwerpunkt auf der Altersgruppe der 30bis 44-jährigen Teilnehmenden (54\%) wurden 635 qualifizierte Beiträge mit 67 konkreten Verbesserungsvorschlägen diskutiert. Die Top-Themenfelder des Dialogs waren die Bereiche Bildung und Betreuung, Wohnumfeld und Verkehr, Freizeit und der Wunsch nach einem zentralen Familieninformationsportal im Internet.

Von Januar bis Mai 2009 führte der Beirat sechs ganztägige Familienforen (4) in den Bezirken, Neukölln, MarzahnHellersdorf, Steglitz-Zehlendorf, Friedrichshain-Kreuzberg, Spandau und Pankow durch. Die Ergebnisse der Foren, die ebenso wie die Gesamtberichterstattung Wert darauf gelegt haben, nicht nur die Schwachpunkte vor Ort zu identifizieren, sondern auch die gute Praxis vorzustellen, wurden in einem Abschlussbericht zusammengestellt und ausgewertet.

Sachverständigenkommission für Achten Familienbericht berufen

Die Bundesministerin für Familie, Senioren, Frauen und Jugend, Kristina Schröder hat eine Sachverständigenkommission mit der Erstellung des Achten Familienberichts bis Sommer 2011 beauftragt. Der Bericht soll unter der Überschrift »Zeit für Verantwortung in der Familie « die Gestaltungsmöglichkeiten für eine gesellschaftliche Zeitpolitik entwickeln, die es Familien besser ermöglichen soll, auch unter veränderten gesellschaftlichen Verhältnissen Verantwortung füreinander zu übernehmen. Die Sachverständigenkommission soll die unterschiedlichen Aspekte von Zeit für Familie untersuchen, den Bedarf von Zeit in Familien ermitteln und auf dieser Grundlage Konzepte für eine familienfreundliche Zeitpolitik entwickeln. Der Kommission gehören folgende Sachverständige an: Dr. Fabienne Becker-Stoll (Staatsinstitut für Frühpädagogik München), Dr. Hans-Peter Klös (Institut der deutschen Wirtschaft Köln), Prof. Dr. Andreas Kruse (Institut für Gerontologie der Universität Heidelberg), Prof. Dr. Dr. h. c. Joachim Möller (Institut für Arbeitsmarkt und Berufsforschung), Prof. Dr. Jutta Rump (Fachhochschule Ludwigshafen), Prof. Dr. Dr. Helmut Schneider (Steinbeis-Hochschule Berlin), Prof. Dr. Norbert Schneider (Bundesinstitut für Bevölkerungsforschung) und Prof. Dr. Gregor Thüsing (Universität Bonn).

Bundesministerium für Familie, Senioren, Frauen und Jugend, Glinkastraße 24, 10117 Berlin, Telefon 03018 555-0, Fax 03018 555-4400, E-Mail poststelle@ bmfsfi.bund.de, Internet http://bmfsfi.de fer (SowiTra) beauftragt, eine Bestandsaufnahme und Bedarfsanalyse (5) zum Thema »Vereinbarkeit von Familie und Beruf im Lebensverlauf von Familien in Berlin« durchzuführen. Der Beirat hat auf der Grundlage dieser Untersuchung konkrete Empfehlungen (6) insbesondere für die Handlungsfelder Arbeitswelt, Kindertagesbetreuung, Informationsangebote und Datenlage formuliert und in einer Deklaration erklärt, wie die Balance zwischen Erwerbsarbeit und Familienleben besser gestaltet und unterstützt werden kann.

\section{Praxisorientierung}

Damit es nicht alleine bei der Expertise bleibt, sucht der Berliner Beirat für Familienfragen auch schon vor Abschluss des Familienberichts nach Möglichkeiten zur Umsetzung in der Praxis. Aus den Empfehlungen des Beirats für eine bessere Vereinbarkeit von Familie und Beruf wurde ein Bündnis des Familienbeirats mit der Industrie- und Handelskammer Berlin, der Handwerkskammer Berlin und dem Deutsche Gewerkschaftsbund, Landesbezirk Berlin-Brandenburg.

Mit Unterzeichnung der »Berliner Deklaration für Vereinbarkeit von Beruf und Familie« (7) haben sich die Partner verpflichtet, einen Schwerpunkt ihrer Aktivitäten der Vereinbarkeit von Familie und Beruf zu widmen. Industrie- und Handelskammer sowie Handwerkskammer haben ihre Betriebsberater entsprechend geschult, der Deutsche Gewerkschaftsbund hat seine Betriebsräte über Vereinbarungsmöglichkeiten informiert. Alle Partner haben gemeinsam den ersten landesweiten Wettbewerb »Unternehmen für Familie - Berlin 2010 « ausgerufen, der im Sommer 2010 die familienfreundlichsten Betriebe Berlins ausgezeichnet hat.

Aus der Forderung des 1. Online-Dialogs nach einem zentralen Informationsportal wurde in Kooperation des Familienbeirats mit dem Verwaltungs- und Organisationsexperten Prof. Dr. Werner Jann an der Universität Potsdam ein studentisches Projekt zur Entwicklung der Inhalte eines Familieninformationsportals. (8) Die Projektergebnisse werden derzeit in einem gemeinsamen Kooperationsprojekt des Familienbeirats mit Prof. Dr. Stephan Breidenbach und der Humboldt Viadrina School of Governance aufgegriffen und praxistauglich umgesetzt. 
Von Mitte Mai bis Ende Juli 2009 hat der Beirat stadtweit einen »Call for Paper « durchgeführt, indem er familienpolitische Akteure eingeladen hatte, BestPractice-Beispiele für mehr Familienfreundlichkeit vorzustellen. So werden praxisrelevante Empfehlungen und gute Beispiele für mehr Familienfreundlichkeit im Familienbericht vorgestellt.

\section{Diskussionsveranstaltungen und Fachforen}

Diskussionsveranstaltungen und Fachforen bieten sich an, um mit Teilnehmenden aus Verbänden, Wissenschaft und Praxis über ausgewählte Themen zu diskutieren. So wurde im April 2009 mit der Friedrich-Ebert-Stiftung die gemeinsame Konferenz »Berlin vernetzen für Familien ...« (9) zum Konzept der Weiterentwicklung der Kindertageseinrichtungen zu Familienzentren veranstaltet. Praxisbeispiele aus Berlin und der gesamten Bundesrepublik haben die Thematik veranschaulicht. Mit der Veranstaltung "Zwischen Pflegebett und Arbeitsplatz « (10) im Februar 2010 hat der Familienbeirat in Kooperation mit den beiden Senatsverwaltungen Familie und Wirtschaft und dem Berliner Lokalen Bündnis für Familie eine Praxisveranstaltung zur Vereinbarkeit von Pflege und Beruf in das öffentliche Interesse der Stadt gerückt.

Im März 2010 wurde ein Hearing zum Thema »Flexible Kindertagesbetreuung in Berlin« durchgeführt. Es ist Grundlage für die Stellungnahme des Familienbeirats zu den Anforderungen an eine flexible Kindertagesbetreuung in der Stadt.

\section{Familie - ein Thema in Berlin!}

Der Berliner Familienbericht 2010 soll am Ende mehr sein, als ein Bericht, der den politischen Entscheidern zeigt, wo nach Ansicht der Experten die Schwachpunkte liegen. Mit der thematischen Konzentration soll deutlich werden, dass ein wichtiger Ausschnitt aus dem breiten Spektrum aller Familienfragen betrachtet wird. Die Verbindung von Expertise mit praktischer Umsetzung macht das Thema schon in der Erarbeitungsphase immer wieder öffentlich. Die Kommunikation der einzelnen Kapitel des Berichts über einen Zeitraum von fast drei Jahren und die Beteiligungselemente in der Berichtserarbeitung haben eine breite Öffentlichkeit in der Stadt erreicht. Damit ist jetzt schon, neben den zukünftigen Empfehlungen für die Politik und dem Handbuchcharakter des Familienberichts Berlin 2010 ein Ziel erreicht: Familie ist Thema in Berlin.

\section{Anmerkungen}

(1) Internet http://www.familienbeiratberlin.de/stellungnahmen.html

(2) Internet http://www.familienbeiratberlin.de/familienbericht.html
(3) Internet http://www.familienbeiratberlin.de/fileadmin/Familienbericht/Abschlussbericht_Berliner_Familiendiskurs_endg.pdf

(4) Internet http://www.familienbeiratberlin.de/fileadmin/Familienbericht/Abschlussbericht_Famforen_en dg.doc.pdf

(5) Bestandsaufnahme und Bedarfsanalyse zum Thema Vereinbarkeit von Familie und Beruf im Lebensverlauf von Familien in Berlin. Internet http://elternzeit-in-berlin.de/Berliner-Familienbericht_Vereinbarkeit_Studie.pdf

(6) Empfehlungen des Berliner Familienbeirats zur Verbesserung der Vereinbarkeit von Familie und Beruf in Berlin. Internet http://www.berlin.de/imperia/md/content/sen-familie/familienpolitik/empfehlungen_familienbeirat_vereinbarkeit_06_2009. pdf? start\&ts $=1247752842 \&$ file $=\mathrm{em}$ pfehlungen_familienbeirat_vereinbarkeit_06_2009.pdf.

(7) Internet http://www.familienbeiratberlin.de/nc/aktuelles/hintergrundinformationen.html?sword_list[]=Deklaration

(8) Internet http://www.familienbeiratberlin.de/fileadmin/Presse/10-0305_Presseeinladung_Familieninformationsportal.pdf

(9) Internet http://library.fes.de/pdf-files/do/06770.pdf

(10) Internet http://www.familienbeiratberlin.de/veranstaltungen/dokumentation.html

\section{Stichwort: Familienberichte der Bundesregierung}

Seit 1965 werden im Auftrag des Bundestages regelmäßig Familienberichte erstellt. Familienberichte werden mindestens in jeder zweiten Legislaturperiode erarbeitet. Eine bis zu siebenköpfige Sachverständigenkommission, die vom Bundesfamilienministerium eingesetzt wird, wird bei der Berichterstellung durch eine Geschäftsführung aus dem Deutschen Jugendinstitut e. V. (DIJ) unterstützt. Dem Familienbericht wird eine Stellungnahme der Bundesregierung angefügt. Familienberichte dienen nicht nur der Politikberatung, wie ursprünglich mit dem Auftrag zur Berichterstattung intendiert, sondern strahlen spätestens seit dem 5. Familienbericht (1994) mit der These der »strukturellen Rücksichtslosigkeit der Gesellschaft gegenüber Familien « auch stark auf die öffentliche und fachpolitische Diskussion zu Familien aus.

Dabei wechseln sich Berichte zur Gesamtsituation von Familien in Deutschland mit denen zu Teilbereichen ab, wie etwa zu "Familien ausländischer Herkunft in Deutschland « (Bundestags-Drucksache 14/4357, 18.10.2000). Mit dem 7. Familienbericht, einem Gesamtbericht zum Thema »Familie zwischen
Flexibilität und Verlässlichkeit - Perspektiven für eine lebenslaufbezogene Familienpolitik « (Bundestags-Drucksache 16/1360, 26.4.2006) sind insbesondere hinsichtlich seiner dialogorientierten Einbindung gesellschaftlicher Gruppen wie Fachöffentlichkeit, Verbänden, Kirchen sowie weiteren Partnern der sogenannten "Allianz für Familie « neue Wege beschritten worden. Im 7. Familienbericht wurden systematisch internationale Erfahrungen einbezogen und Zukunftsszenarien zur alltäglichen und lebenslaufbezogenen Zeitpolitik mit einem Plädoyer für »Optionszeiten «, zu kommunalen Infrastrukturen, zu Familienkasse und Elterngeld sowie zu verstärkter interdisziplinärer Familienforschung entwickelt.

Karin Jurczyk

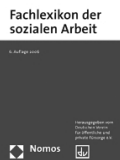

Quelle: Deutscher Verein für öffentliche und private Fürsorge e. V. (Hg.): Fachlexikon der sozialen Arbeit. 6. Auflage. Nomos Verlagsgesellschaft, Baden-Baden 2007. 1.195 Seiten. 44,- Euro. ISBN 978-3-8329-1825-5. Seite 312. 